\title{
Perfil de Atendimento em Unidades de Pronto Atendimento (UPAs) em um município do oeste paranaense, segundo o Protocolo de Manchester
}

\author{
Profile Attendance at treated for Emergency Care Units (UPAs) in a municipality in western
}

Paraná, according to the Manchester Protocol

Perfil de Asistencia en las Unidades de Atención de Emergencias (UPAs) de una ciudad del oeste de Paraná, según el Protocolo de Manchester

Recebido: 05/07/2021 | Revisado: 13/07/2021 | Aceito: 14/07/2021 | Publicado: 24/07/2021

\author{
Thainá Klosowski Kulicz \\ ORCID: https://orcid.org/0000-0002-6125-1378 \\ Centro Universitário FAG, Brasil \\ E-mail: thainakulicz2@hotmail.com \\ Kurt Juliano Sack Orejuela Uscocovich \\ ORCID: https://orcid.org/0000-0002-6119-6700 \\ Centro Universitário FAG, Brasil \\ E-mail: kurtsou@yahoo.com.br
}

\begin{abstract}
Resumo
As Unidades de Pronto Atendimento (UPAs) que têm por finalidade prestar atendimentos de média complexidade estão se tornando uma porta de entrada para resolução de atendimentos de baixa complexidade. O presente estudo avaliou o perfil de atendimento de pacientes que procuram as UPAs em um município do oeste do Paraná, segundo o Protocolo de Manchester. Com isso, objetivou-se caracterizar a demanda de usuários que buscam suporte na Atenção Secundária e identificar se o uso desses serviços ocorre conforme é determinado pela Política Nacional de Atenção às Urgências e Emergências (PNAU). Trata-se de uma pesquisa retrospectiva e transversal de abordagem quantitativa. Para determinação de resultados foram selecionadas pessoas, de todas as idades, atendidas em 2 UPAs do município durante o período de janeiro até dezembro de 2019. A amostra válida do estudo foi de 144.459 atendimentos realizados e classificados de acordo com a classificação de risco. Constatou-se que a maioria dos atendimentos prestados foram classificados como verde e amarelo, diferentemente do que é proposto pela lei. Portanto, concluiu-se que existe uma divergência entre a proposta original de que atendimentos de média complexidade devem ser prestados pelas UPAs, como estabelecido pela PNAU, e o atual cenário de atendimentos realizado por essas Unidades. Palavras-chave: Protocolos; Serviços de atendimento de emergência; Triagem; Níveis de atenção à saúde; Atenção secundária à saúde.
\end{abstract}

\begin{abstract}
The Emergency Care Units (UPAs) whose purpose is to provide medium-complexity care are becoming a gateway to the resolution of low-complexity care. This study evaluated the profile of care provided to patients treated for UPAs in a municipality in western Paraná, according to the Manchester Protocol. The objective was to characterize the demand of users who seek assistance in Secondary Care and to identify whether the use of these services occurs as determined by the National Policy for Attention to Urgencies and Emergencies. It is a retrospective and transversal research with a quantitative approach. To determine the results, people of all ages, served in 2 UPAs in the municipality from January to December 2019 were selected. The valid sample of the study was 144,459 visits made and classified according to the risk classification. It was found that most of the services provided were classified as green and yellow, differently from what is proposed by law. Therefore, it was concluded that there is a divergence between the original proposal that medium complexity care should be provided by UPAs, as established by PNAU, and the current scenario of care in these Units.
\end{abstract}

Keywords: Protocols; Emergency medical services; Triage; Health care levels; Secondary care.

\section{Resumen}

Las Unidades de Atención de Emergencias (UPAs), cuya finalidad es atender la complejidad media, se están convirtiendo en una puerta de entrada a la resolución de la atención de baja complejidad. Este estudio evaluó el perfil de atención de los pacientes que acuden as UPAs en una ciudad del oeste de Paraná, según el Protocolo de Manchester. El objetivo fue caracterizar la demanda de los usuarios que buscan asistencia en Atención Secundaria e identificar si el uso de estos servicios se da según lo determinado por la Política Nacional de Atención de Urgencias y Emergencias (PNAU). Se trata de un estudio retrospectivo, transversal con enfoque cuantitativo. Para determinar los 
resultados se seleccionaron personas de todas las edades, atendidas en 2 UPA de la ciudad durante el período de enero a diciembre de 2019. La muestra válida del estudio fue de 144.459 consultas realizadas y clasificadas según la clasificación de riesgo. Se encontró que la mayoría de los servicios prestados fueron clasificados como verde y amarillo, a diferencia de lo que propone la ley. Por lo tanto, se concluyó que existe una divergencia entre la propuesta original de que la atención de complejidad media debe ser brindada por las UPA, según establece el PNAU, y el escenario actual de atención en estas Unidades.

Palabras clave: Protocolos; Servicios médicos de urgencia; Triaje; Niveles de atención de salud; Atención secundaria de salud.

\section{Introdução}

O Sistema Único de Saúde (SUS) é composto por uma série de serviços articulados que se complementam e tem como objetivo atender de maneira eficiente toda a população que necessita de suporte (Brasil, 1990). Os serviços oferecidos pelo SUS são separados em níveis de atenção, sendo elas primária, secundária e terciária. Essa estrutura visa organizar as ações e serviços oferecidos pelo sistema (Brasil, 1990; Brasil, 2011).

A Atenção Primária à Saúde, preferencial porta de entrada ao SUS, é composta pelas Unidades Básicas de Saúde (UBS) e Unidades de Saúde da Família (USF). Sua função é realizar atendimentos de baixo risco e também atuar na promoção, prevenção, diagnóstico e tratamento precoce dos pacientes. É essencial fazer um acompanhamento contínuo desses pacientes afim de evitar futuras complicações preveníveis (Brasil, 2017). Esse nível de complexidade é capaz de atender e resolver mais de $80 \%$ dos problemas de saúde da população (Paim, 2019).

Os serviços de média complexidade são realizados por estabelecimentos intermediários entre as UBS e a Rede Hospitalar, as Unidades de Pronto Atendimento (UPAs). São, basicamente, hospitais simplificados que funcionam 24 horas por dia em todos os dias da semana. $\mathrm{O}$ acolhimento dos pacientes é realizado com base na classificação de risco o que permite rápido suporte àqueles que necessitam de conduta imediata. Portanto, o que dita a priorização do atendimento é a gravidade do caso e não a ordem de chegada a Unidade (Brasil, 2017; Brasil, 2011). Atualmente, o Sistema de Triagem de Manchester (STM) é o protocolo de classificação por cor mais utilizado nas UPAs do Brasil. Essas estruturas são regidas pela Política Nacional de Urgências e Emergências (PNAU), publicada pelo Ministério da Saúde em 2003 (Brasil, 2011).

A alta complexidade é designada à Atenção Hospitalar que proporciona uma série de terapias e procedimentos altamente especializados, além de envolverem alta tecnologia (Brasil, 2011).

A participação da população é um dos principais pilares para o funcionamento adequado do SUS (Brasil, 1990). É importante que os usuários entendam o sistema, saibam a função de cada complexidade de saúde, conheçam o acolhimento com classificação de risco e façam o uso correto dos serviços oferecidos para que não ocorra a desarticulação dos diferentes níveis de atenção (Tostes, et al., 2017).

Na prática, a grande maioria dos utilizadores do sistema desconhecem a organização e o funcionamento do SUS (Hamada, et al., 2018; Kawashima, et al., 2016). Devido a essa falta de informação e concepção (Tostes, et al., 2017; Hamada, et al., 2018; Kawashima, et al., 2016), é observado que as pessoas não seguem a hierarquização dos serviços de saúde de acordo com a complexidade do atendimento, como é estabelecido pela lei (Brasil, 2011).

Mesmo com a ampliação da oferta de serviços de Atenção Básica desde 1990, é comum ver grande parte da população em busca de consultas não urgentes na Atenção Secundária (Machado, Salvador, O'Dwyer, 2011). Isso dificulta o atendimento integral ao paciente, tornando a assistência fragmentada.

As UPAs estão sendo a escolha, como porta de entrada ao sistema, para solucionarem problemas de saúde sensíveis de serem resolvidos pela Atenção Primária (Gomide, Pinto, Figueiredo, 2012; Caccia-Bava, et al., 2011; Cassettari, Mello, 2017; Oliveira, 2019; Oliveira, et al., 2011). Os pacientes procuram essas Unidades pela rápida e fácil resolução dos seus problemas, além delas oferecem mais recursos e opções de exames complementares (Marques, Lima, 2007). Como 
consequência desse uso equivocado da Atenção Secundária como o primeiro acesso aos serviços de saúde é notado a superlotação nessa complexidade, falta de leitos, aumento das filas de espera e estresse tanto profissional quanto do usuário (Siqueira, 2010).

Neste sentido, devido a importância do reconhecimento do uso inadequado desses serviços, o estudo analisou o perfil de atendimento, de acordo com o Protocolo de Manchester, realizado nas UPAs de um município do oeste paranaense. O presente artigo se propõe a caracterizar a demanda de pacientes que buscam suporte na Atenção Secundária e identificar se o uso desses serviços ocorre conforme determinado pela Política Nacional de Atenção às Urgências e Emergências (PNAU).

\section{Metodologia}

Este estudo foi baseado metodologicamente como uma pesquisa retrospectiva e transversal de abordagem quantitativa.

Os dados foram coletados através da análise de prontuários eletrônicos fornecidos pelo sistema de informação, IPM Saúde, utilizado pela Secretária Municipal de Saúde do munícipio. O IPM Saúde é um software que permite o armazenamento de informações de saúde em uma única plataforma. Nele é possível ter acesso a identificação de pacientes, prontuários eletrônicos e classificação de atendimento de acordo com a gravidade de cada caso. Por meio deste, obteve-se acesso ao fluxo de atendimentos prestados pelas UPAs, conforme a classificação de risco realizado na triagem. Os dados foram analisados e organizados em tabelas no Microsoft Excel que possibilitou a construção do gráfico apresentado no trabalho. A população de estudo foi composta por pessoas, de todas as idades, que buscaram atendimento em 2 UPAs de um município do oeste do Paraná e foram classificadas em sua admissão conforme o Protocolo de Manchester, no período de 1 de janeiro de 2019 até 31 de dezembro 2019.

O Sistema de triagem de Manchester (STM), criado em 1994 na Inglaterra e implantado pela primeira vez no Brasil em 2008, classifica a gravidade do caso do paciente e determina o tempo máximo que o usuário pode aguardar pelo atendimento (Coutinho, Cecílio, Mota, 2012; Mackway-Jones, Marsden, Windle, 2014). Sua classificação permite a avaliação dos pacientes por meio dos critérios clínicos e não pela ordem de chegada ao serviço. O protocolo é composto por 5 níveis de prioridade: nível 1 (vermelho) são pacientes emergentes de cuidado e devem receber atendimento médico imediato; nível 2 (laranja) são pacientes muito urgentes e precisam de atendimento em até 10 minutos; nível 3 (amarelo) são pacientes urgentes e necessitam de avaliação médica em até 60 minutos; nível 4 (verde) são pacientes pouco urgentes que devem ser atendidos em até 120 minutos e, por fim, nível 5 (azul) são os pacientes não urgentes e que podem esperar o atendimento até 240 minutos (Mackway-Jones, Marsden, Windle, 2014).

O Projeto de Pesquisa foi submetido ao Comitê de Ética em Pesquisa com Seres Humanos do Centro Universitário FAG e aprovado dia 29 de fevereiro de 2020 pelo CAAE número 26536719.1.0000.5219. Por se tratar de uma pesquisa com seres humanos, este estudo está em cumprimento com a Resolução 466/12 do Conselho Nacional de Saúde.

\section{Resultados}

Em uma observação geral, foram registrados 144.459 atendimentos totais nas UPAs Brasília e Veneza do município de Cascavel-PR durante o período de 1 de janeiro de 2019 até 31 de dezembro de 2019.

Quando dividimos conforme a classificação de risco, a maior parte dos pacientes foi classificado na categoria verde ou pouco urgente, correspondendo a 101.302 atendimentos (70,12\%), seguido da cor amarelo ou urgente com 36.047 (24,95\%), azul com 4.252 (2,94\%), laranja com $2.756(1,90 \%)$ e, por fim, a cor vermelha ou emergente com 102 atendimentos $(0,07 \%)$. 
Em uma análise isolada, foram 68.066 atendimentos na UPA Brasília e 76.393 atendimentos na UPA Veneza. Quanto a classificação de risco, foram verificados na UPA Brasília 47.945 atendimentos verdes, 16.073 amarelos, 2.788 azuis, 1.223 laranjas e 37 vermelhos. Já na UPA Veneza foram contabilizadas 53.357 consultas verdes, 19.974 amarelos, 1.464 azuis, 1.533 laranjas e 65 vermelhos. Os resultados encontram-se no Gráfico 1.

Gráfico 1: Atendimentos conforme classificação de risco realizados no período de janeiro até dezembro de 2019 nas UPAs Brasília e Veneza de Cascavel-PR.

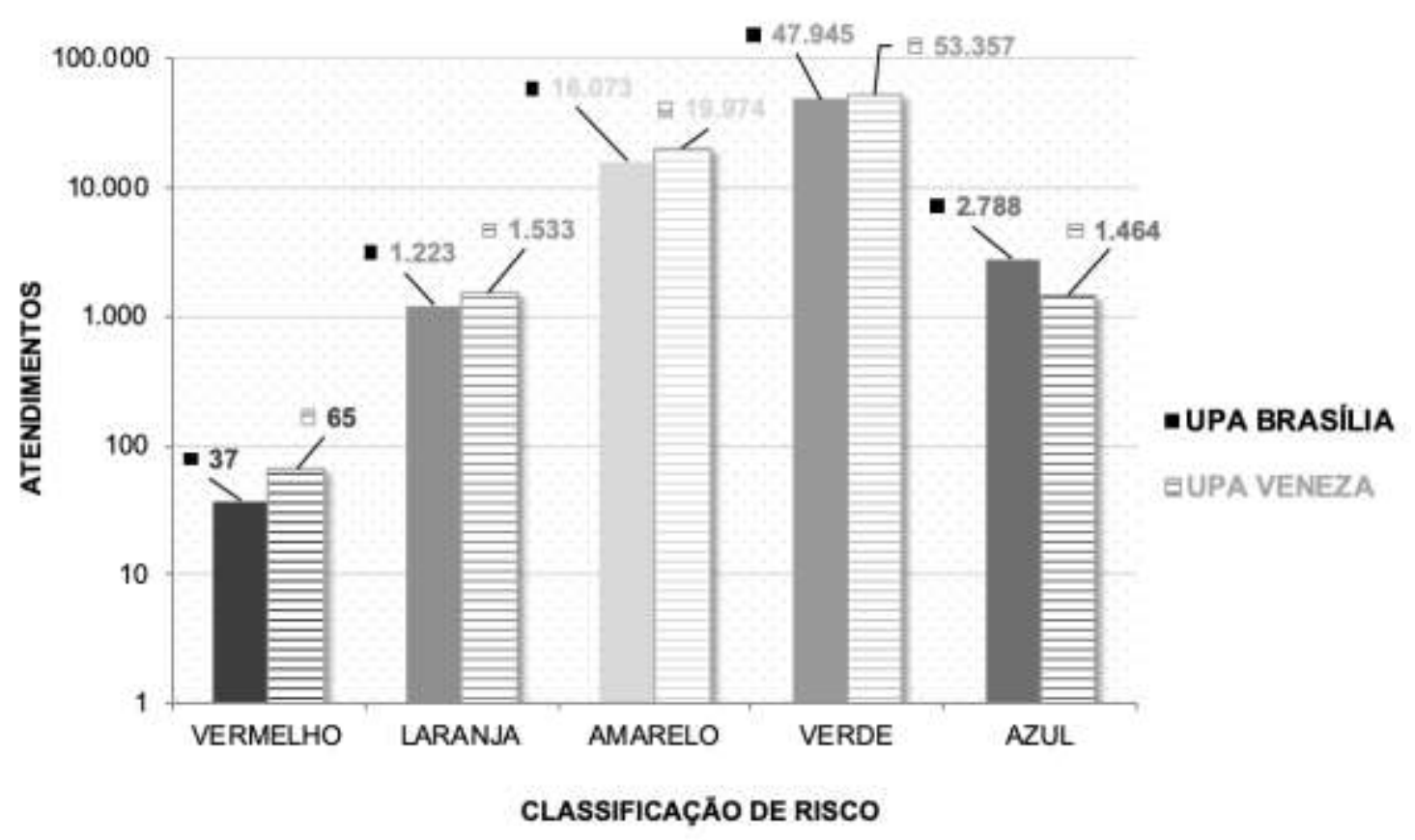

Fonte: Autores (2020).

\section{Discussão}

Existe uma divergência entre a proposta original de que atendimentos de média complexidade devem ser prestados pelas UPAs, como estabelecido pela PNAU, e o atual cenário de atendimentos realizado por essas Unidades.

As UPAs têm como propósito acolher pacientes em situação de urgência e emergência, prestar atendimento resolutivo e qualificado aos quadros agudos ou crônicos agudizados de natureza clínica e prestar o primeiro suporte aos casos de natureza cirúrgica e de trauma (Brasil, 2011). Apesar da especificidade destes serviços para acolher os casos de saúde de média gravidade, a realidade é marcada pela inversão de fluxo de usuários entre a Atenção Básica e a Atenção Secundária.

As UPAs do munícipio estudado seguem o acolhimento conforme protocolo de Manchester, no qual os casos classificados como emergência (vermelho) necessitam de atendimento imediato; muito urgente (laranja) devem ser atendidos em até 10 minutos; urgente (amarelo) podem ser atendidos em até 60 minutos; pouco urgentes podem aguardar atendimento por até 120 minutos e não urgentes que podem aguardar atendimento por até 240 minutos (Coutinho, Cecílio, Mota, 2012). Observou-se que apenas $0,07 \%$ dos usuários foram classificados como emergência (vermelho) e 24,95\% como urgência (amarelo), mostrando uma distorção do verdadeiro papel das UPAs, o qual seria de atender urgências e emergências.

Entre os pacientes pesquisados evidenciou-se que houve mais atendimentos à pessoas classificadas como verde (70,12\%), este dado se assemelha ao de outros estudos realizados por pesquisadores brasileiros (Oliveira, 2019; Oliveira, et al., 2011). Em estudos semelhantes, obtiveram como resultado que $67 \%$ dos atendimentos em uma Unidade de emergência 
referenciada de um hospital de ensino (Oliveira, 2019) e 51\% dos atendimentos prestados na UPA de outro munícipio avaliado (Oliveira, et al., 2011) foram classificados como verde. Os autores associam esta alta demanda de atendimentos pouco urgentes a uma falta de orientação da população sobre a utilização correta dos serviços disponíveis e a uma carência da concepção concreta do que seriam casos de urgência e emergência.

Portanto, os resultados apresentados corroboram a hipótese desse estudo de que o serviço prestado pelas UPAs não está diretamente relacionado à sua proposta original e também sustenta as pesquisas realizadas por outros pesquisadores (Gomide, Pinto, Figueiredo, 2012; Caccia-Bava, et al., 2011; Cassetari, Melo, 2017; Oliveira, 2019; Oliveira, et al., 2011) que mostraram que grande parte dos atendimentos realizados pelas UPAs são de baixa complexidade.

Os Órgãos Públicos não conseguem informar sobre os serviços ofertados e o caminho que o usuário deve percorrer dentro do sistema (Hamada, et al., 2018). A falta de conhecimento da população (Tostes, et al., 2017; Hamada, et al., 2018; Kawashima, et al., 2016; Oliveira, 2019; Oliveira, et al., 2011) é um sério fator que gera a desarticulação dos níveis de atenção a saúde. Por não saberem como o SUS funciona, os usuários dirigem-se ao serviço de saúde que mais os convêm.

Uma revisão sistemática realizada para compreender as razões que levam o paciente a buscar o serviço de Pronto Atendimento (PA), quando estes poderiam ser atendidos na Atenção Básica, revelou que a restrição de horário de funcionamento das UBS, a dificuldade de encontrar agenda disponível na UBS, considerar que sua doença necessita de um atendimento de urgência e a maior disponibilidade de recursos no PA do que na UBS são fatores que influenciam a decisão de procurar por este tipo de serviço. Dessa forma, é evidente que os usuários preferem a rápida resolução dos seus problemas e por isso dirigem-se aos PA (Caccia-Bava, et al., 2011; Dixe, et al., 2018; Marques, Lima, 2007; Schafirowitz, de Souza, 2020).

O uso errôneo dos serviços da Atenção Secundária é preocupante, uma vez que essa busca crescente por consultas causa uma demanda maior do que a capacidade de atendimentos e, consequentemente, retrata um cenário de superlotação e falta de leitos nas UPAs (Siqueira, 2010; Azevedo, et al., 2010). Além disso, há a desorganização das Unidades, elevação das despesas ao sistema e geração de gastos desnecessários, o que resulta em uso pouco racional dos recursos disponíveis (Azevedo, et al., 2010).

Outro problema que ocorre é um atendimento médico que não consegue estabelecer vínculo dos usuários com os serviços, fator fundamental para garantir cuidados contínuos em saúde. Naquele momento, os pacientes só recebem tratamento para aliviar sintomas pontuais e esquecem da importância de um acompanhamento médico para prevenir novas complicações e outras doenças (Stein, et al., 2002).

A nossa sociedade requer mudanças culturais. As pessoas precisam entender a estrutura hierárquica dos serviços médicos, a importância da articulação dos níveis de saúde e a importância do acompanhamento contínuo (Oliveira, 2019).

A partir dos achados do presente estudo, é importante traçar metas para melhorar os serviços. Sugere-se a implementação de campanhas e políticas educacionais direcionadas à população com o propósito de orientá-los sobre o SUS, a articulação dos diferentes níveis de complexidade do sistema, o conceito da classificação de risco e como usar de maneira correta os serviços oferecidos.

\section{Conclusão}

O presente estudo permitiu evidenciar que grande parte dos atendimentos prestados pelas UPAs avaliadas não necessitavam dos serviços de Atenção Secundária e, em sua maioria, poderiam ser resolvidos pela Atenção Básica. Constatouse que a maioria dos atendidos foram classificados, de acordo com o Protocolo de Manchester, como verde e amarelo que são considerados, respectivamente, pacientes pouco urgentes e urgentes. Contudo, vale lembrar que as UPAs foram criadas com objetivo de atender situações de urgência e emergência. 
Na perspectiva da consolidação dos princípios do SUS, é importante compreender essa realidade em nosso meio no sentido de avaliar as atuais necessidades de reorganização do sistema de saúde. Definir a demanda e o perfil da população que procura a Atenção Secundária, proporciona o reconhecimento dos usos inadequados do serviço e a necessidade de reprimi-los, a fim de evitar superlotação das Unidades e estresse profissional e do paciente.

Como limitação deste estudo podemos referir o fato do método de triagem, Protocolo de Manchester, usado nas UPAs ser operador dependente, o que pode interferir na classificação de risco de cada caso. A abordagem de apenas 2 Unidades de Pronto Atendimento também pode ser considerada um fator limitante tendo em vista que o tamanho da amostra é pequeno em relação a quantidade de unidades existentes pelo Brasil.

Esta pesquisa abre espaço para futuros artigos científicos longitudinais abordarem sobre este tema para esclarecer aos usuários sobre o SUS, a função de cada complexidade de saúde, o conceito da classificação de risco e, mais importante, como fazer o uso correto dos serviços oferecidos. Sugere-se a elaboração de campanhas e políticas educacionais direcionadas à população cascavelense com o intuito de informá-los e instruí-los.

\section{Referências}

Azevedo, A. L. D. C. S., Pereira, A. P., Lemos, C., Coelho, M. F., \& Chaves, L. D. P. (2010). Organização de serviços de emergência hospitalar: uma revisão integrativa de pesquisas. Revista Eletrônica de Enfermagem, 12(4), 736-45. https://doi.org/10.5216/ree.v12i4.6585

Brasil. Decreto ${ }^{\circ}$ 7.508, de 28 de junho de 2011. Regulamenta a Lei n 8.080 , de 19 de setembro de 1990, para dispor sobre a organização do Sistema Único de Saúde - SUS, o planejamento da saúde, a assistência à saúde e a articulação interfederativa, e dá outras providências. Diário Oficial da União, Brasília, 2011. http://www.planalto.gov.br/ccivil_03/_ato2011-2014/2011/decreto/d7508.htm.

Brasil. Lei $\mathrm{n}^{\circ}$ 8.080, de 19 de setembro de 1990. Dispõe sobre as condições para a promoção, proteção e recuperação da saúde, a organização e o funcionamento dos serviços correspondentes e dá outras providências. Brasília: Diário Oficial da União, 1990. http://www.planalto.gov.br/ccivil_03/leis/18080.htm.

Brasil. Portaria $\mathrm{n}^{\mathrm{o}}$ 10, de 3 de janeiro de 2017. Redefine as diretrizes de modelo assistencial e financiamento de UPA 24h de Pronto Atendimento como Componente da Rede de Atenção às Urgências, no âmbito do Sistema Único de Saúde. Ministério da Saúde, Brasília, 2017. http://bvsms.saude.gov.br/bvs/saudelegis/gm/2017/prt0010_03_01_2017.html.

Brasil. Portaria $n^{\circ}$ 1.600, de 7 de julho de 2011. Reformula a Política Nacional de Atenção às Urgências no Sistema Único de Saúde (SUS). Ministério da Saúde, Brasília, 2011. http://bvsms.saude.gov.br/bvs/saudelegis/gm/2011/prt1600_07_07_2011.html.

Brasil. Portaria n 2.436, de 21 de setembro de 2017. Aprova a Política Nacional de Atenção Básica, estabelecendo a revisão de diretrizes para a organização da Atenção Básica, no âmbito do Sistema Único de Saúde (SUS). Ministério da Saúde, Brasília, 2017. https://bvsms.saude.gov.br/bvs/saudelegis/gm/2017/prt2436_22_09_2017.html.

Caccia-Bava, M.C.G., Pereira, M. J. B., Rocha, J. S. Y., \& Martinez, E. Z. (2011). Pronto-atendimento ou atenção básica: escolhas dos pacientes no SUS. Medicina (Ribeirão Preto), 44(4), 347-354. https://doi.org/10.11606/issn.2176-7262.v44i4p347-354

Cassettari, S. D. S. R., \& Mello, A. L. S. F. D. (2017). Demanda e tipo de atendimento realizado em unidades de pronto atendimento do município de Florianópolis, Brasil. Texto \& Contexto-Enfermagem, 26. https://doi.org/10.1590/0104-07072017003400015

Coutinho, A. A. P., Cecílio, L. C. D. O., \& Mota, J. A. C. (2012). Classificação de risco em serviços de emergência: uma discussão da literatura sobre o Sistema de Triagem de Manchester. Rev Med Minas Gerais, 22(2), 188-98.

Dixe, M. D. A. C. R., Passadouro, R., Peralta, T., Ferreira, C., Lourenço, G., \& de Sousa, P. M. L. (2018). Determinantes do acesso ao serviço de urgência por utentes não urgentes. Revista de Enfermagem Referência, 4(16), 41-51. https://doi.org/10.12707/ RIV17095

Gomide, M. F. S., Pinto, I. C., \& Figueiredo, L. A. D. (2012). Acessibilidade e demanda em uma Unidade de Pronto Atendimento: perspectiva do usuário. Acta Paulista de Enfermagem, 25, 19-25. https://doi.org/10.1590/S0103-21002012000900004

Hamada, R. K. F., de Almeida, V. O. C., Brasil, I. C. F., Souza, S. G., Luzia, R. A. B., Campos, E. M. S., \& Leite, I. C. G. (2018). Conhecendo o Sistema Único de Saúde: um olhar da população. Revista de APS, 21(4).

Kawashima, M. D. S., Ferreira, M. V., Silva, P. D., Boaventura, A., \& Almeida, J. D. (2016). O Desconhecido SUS: O conhecimento da população de uma cidade do Vale do Paraíba. Universidade do Vale do Paraíba. http://www.inicepg.univap.br/cd/INIC_2007/trabalhos/saude/inic/INICG00420_01O.pdf.

Machado, C. V., Salvador, F. G. F., \& O'Dwyer, G. (2011). Serviço de Atendimento Móvel de Urgência: análise da política brasileira. Revista de Saúde Pública, 45, 519-528.

Mackway-Jones, K., Marsden, J., \& Windle, J. (Eds.). (2014). Emergency triage: Manchester triage group. John Wiley \& Sons.

Marques, G. Q., \& Lima, M. A. D. D. S. (2007). Demandas de usuários a um serviço de pronto atendimento e seu acolhimento ao sistema de saúde. Revista Latino-americana de enfermagem, 15, 13-19. https://doi.org/10.1590/S0104-11692007000100003 
Research, Society and Development, v. 10, n. 9, e21610917910, 2021

(CC BY 4.0) | ISSN 2525-3409 | DOI: http://dx.doi.org/10.33448/rsd-v10i9.17910

Oliveira, G. N., Silva, M. D. F. N., Araujo, I. E. M., \& Carvalho-Filho, M. A. (2011). Perfil de la población atendida en una unidad de emergencia de referencia. Revista Latino-Americana de Enfermagem, 19, 548-556. https://doi.org/10.1590/S0104-11692011000300014

Oliveira, M. D. D. (2019). Perfil da demanda de atendimentos realizados pela Unidade de Pronto Atendimento (UPA) de Tramandaí/RS. http://hdl.handle.net/10183/201878

Paim, J. (2009). O que é o SUS. SciELO-Editora FIOCRUZ.

Schafirowitz, G. D. C., de Souza, A. C. (2020). Usuários adultos classificados como pouco urgentes em Unidade de Pronto Atendimento. InterfaceComunicação, Saúde, Educação, 24. https://doi.org/10.1590/Interface.190630

Siqueira, I. L. C. P. (2010). Qualidade em serviço de emergência. Sallum AMC, Paranhos WY. O enfermeiro e as situações de emergência. São Paulo (SP): Atheneu, 25-34.

Stein, A. T., Harzheim, E., Costa, M., Busnello, E., \& Rodrigues, L. C. (2002). The relevance of continuity of care: a solution for the chaos in the emergency services. Family practice, 19(2), 207-210. https://doi.org/10.1093/fampra/19.2.207

Tostes, P. C. R., Celestino, C. S. M., da Cruz, K. M., \& Chevitarese, L. (2017). Acolhimento e classificação de risco em unidade de pronto atendimento (UPA): uma abordagem educativa. Revista Rede de Cuidados em Saúde, 10(1). http://publicacoes.unigranrio.edu.br/index.php/rcs/article/view/3289 